\title{
UAV ATTITUDE ESTIMATION BY COMBINING HORIZON-BASED AND HOMOGRAPHY-BASED APPROACHES FOR CATADIOPTRIC IMAGES
}

\author{
Jean-Charles Bazin *, InSo Kweon* \\ Cédric Demonceaux ${ }^{* *}$, Pascal Vasseur ${ }^{* *}$ \\ ${ }^{*}$ RCV Lab, KAIST, Korea \\ ** CREA, UPJV, France
}

\begin{abstract}
Developing Unmanned Aerial Vehicles (UAV) with self-stabilization capabilities represents an intensive research field nowadays. This paper aims to show that combining our previous horizon extraction algorithm with homography methods permits to estimate the homography more robustly and thus the UAV attitude. We show that imposing horizon constraint permits to remove evident inconvenient non-planar points. Moreover, we explain that computing the horizon in the sphere permits to compute the normal vector of the ground plane and thus retrieve the right motion among the four possible solutions obtained by planar homography, which is not a trivial problem usually.
\end{abstract}

Keywords: catadioptric vision, UAV, homography, horizon, pose estimation

\section{INTRODUCTION}

A key requirement for the autonomy of UAVs is their stabilization. Diverse experiments have shown that traditional navigations devices such as Inertial Navigation System or Global Positioning System are far from being perfect equipments. For instance, INS accumulates measurement error over time and needs to be periodically reset. Moreover it is sensitive to vibrations which is common in helicopter applications. The limitation of GPS is that signal can be lost (in a tunnel, in Tokyo downtown, etc...) and measurements can be unreliable due to possible hostile jamming. As an alternative to these systems, some works have proposed a vision-based approach of the UAV navigation problem (Naval et al., 1997),(Rushant and Spacek, 1997)(Wang et al., 2005)(Woo and Kweon, 2005). Most of them use conventional

\footnotetext{
1 This work was partially supported by GIP-ANR
} CaViAR project cameras which have a relative small field of view, which drastically limits the information we could get from the environment (cf Fig. 1). Therefore we have previously proposed a method based on catadioptric vision which provides a very wide field of view and we have been able to estimate the roll and pitch angles after extracting the horizon in catadioptric images (Demonceaux and Vasseur, 2006). In order to estimate the three rotation angles, there exist some epipolar techniques, such as planar homography or fundamental matrix, that estimate the motion from point correspondences between two images. However in catadioptric aerial images, many features points may lie on the horizon or in the sky. All these points do not verify the planar homography relation since they lie at infinity. Indeed they are invariant to translation and dependent on rotation. The idea is to remove these inconvenient points by simply imposing horizon constraint based on our previous horizon extraction algorithm. It will lead 
to a more accurate estimation of the homography and thus of the UAV attitude. Moreover, we show that computing the horizon in the sphere permits to compute the normal vector of the ground plane and thus retrieve the right motion among the four possible solutions obtained by planar homography, which is not a trivial problem usually.
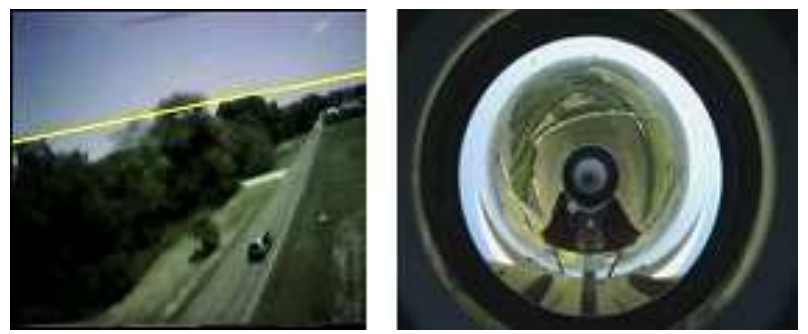

Fig. 1. compared to traditional cameras (left from( Ettinger and al, 2002)), catadioptric system (right) can gather much more information from the environment, such as a much larger part of the horizon.

\section{CATADIOPTRIC VISION AND IMAGE FORMATION}

Traditional cameras have a small field of view: they generally cover about only 50 degrees of the whole scene. As a consequence, such cameras can perceive only a small portion of the horizon line. This portion is still more unreliable if many obstacles, such as trees or buildings, obstruct the horizon. Therefore every horizon-based algorithm for attitude estimation, such as (Ettinger et al., 2003), cannot correctly perform in such situations. Similarly, the images taken by narrow field of view cameras can contain large textureless regions (for example homogeneous wall or floors) and as a consequence, methods using points correspondence for epipolar constaint, such as (Wang et al., 2005), are likely to fail. Intuitively, if the field of view is wider, we can gather much more information from the environment and thus the pose estimation will be more accurate. For this purpose, catadioptric cameras have been developed. They belong to the family of omnidirectional systems, i.e. which can see in all directions, and are composed of a mirror with a specific shape and a conventional camera. Thanks to the mirror projection, they can perceive the scene at 360 degrees in the horizontal plane and more than 90 degrees in the vertical plane. In (Geyer and Daniilidis, 2001), the authors have sown that a central catadioptric projection is equivalent to a two step mapping via a sphere: first a point $X_{w}$ in $3 \mathrm{D}$ world is projected to a unit sphere at $X_{s}$ with respect to the single effective viewpoint $O$, then the point $X_{s}$ on the sphere is perceptively projected to the image plane at $\mathrm{m}$ from a particular point $O_{c}$, obtained by calibration (cf

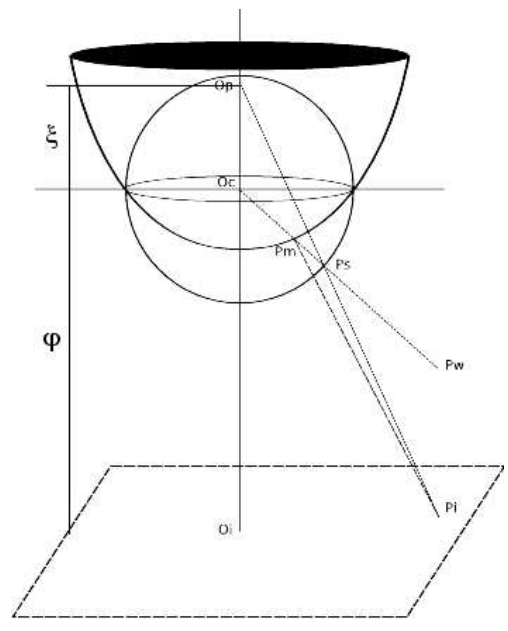

Fig. 2. Equivalence between the catadioptric projection and the two-step mapping via the sphere (cf. text for details)

Fig 2. This point $m$ would have been the same if we had performed the traditional catadioptric projection, meaning on the mirror and then on the image plane. In the more general approach of (Barreto, 2006), it is proved that projections involved in central catadioptric systems, perspective cameras and devices with lens distortion can all be unified in the single sphere representation. This sphere projection theorem has strong implications in UAV pose estimation, as show in the following sections.

\section{HOMOGRAPHY FOR CATADIOPTRIC VISION}

Epipolar geometry is the intrinsic projective geometry between two views and is related to two important tools among others: planar homography $\mathrm{H}$ and fundamental matrix $F$ (Hartley and Zisserman, 2004) (Faugeras, 1993). They both permit to estimate the camera motion between a pair of images from point correspondences. Whereas fundamental matrix can be applied to general 3D scenes, planar homography is dedicated to planar scenes. In our studied application, the UAV is flying at relative high altitude and the ground in the image is considered plane since the earth curvature can be intuitively neglected. Therefore the planar homography is preferred to the more general fundamental matrix.

Let $P$ a world point whose coordinates are $\left(x_{w}, y_{w}, z_{w}\right)^{T}$ in the frame associated to the first image and is projected onto the associated sphere at $\left(x_{s}, y_{s}, z_{s}\right)^{T}=\lambda\left(x_{w}, y_{w}, z_{w}\right)^{T}$ where $\lambda=$ $\frac{1}{\sqrt{x_{w}^{2}+y_{w}^{2}+z_{w}^{2}}}$. Respectively, $P$ is represented by $\left(x_{w}^{\prime}, y_{w}^{\prime}, z_{w}^{\prime}\right)^{T}$ in the frame associated to the second image and is projected onto the associated sphere at $\left.\left(x_{s}^{\prime}, y_{s}^{\prime}, z_{s}^{\prime}\right)^{T}\right)=\lambda^{\prime}\left(x_{w}^{\prime}, y_{w}^{\prime}, z_{w}^{\prime}\right)^{T}$ where $\lambda^{\prime}=\frac{1}{\sqrt{x_{w}^{\prime 2}+y_{w}^{\prime 2}+z_{w}^{\prime 2}}}$. While there exists a very 
large literature about homography for traditional perspective cameras, homography for catadioptric images has been introduced only recently (Benhimane and Malis, 2006) (fig 3) and is defined as below:

$$
\left(\begin{array}{l}
x_{s}^{\prime} \\
y_{s}^{\prime} \\
z_{s}^{\prime}
\end{array}\right)=\frac{\lambda^{\prime}}{\lambda} H\left(\begin{array}{l}
x_{s} \\
y_{s} \\
z_{s}
\end{array}\right)
$$

where

$$
H=R+T \tilde{n}^{T}
$$

$\tilde{n}$ is the normal to the plane such that $\tilde{n}=\frac{n}{d}$ where $d$ is the distance from the center of the sphere. Matrix $H$ has 8 degrees of freedom and each point correspondence provides two sets of equations. Therefore at least four pairs of points are required to compute $H$ up to scale. Solving $H$ from point correspondence can be performed by minimizing either the algebraic distance by linear methods (typically SVD) or the geometric reprojection error which involves non-linear optimization but provides improved results (Hartley and Zisserman, 2004).

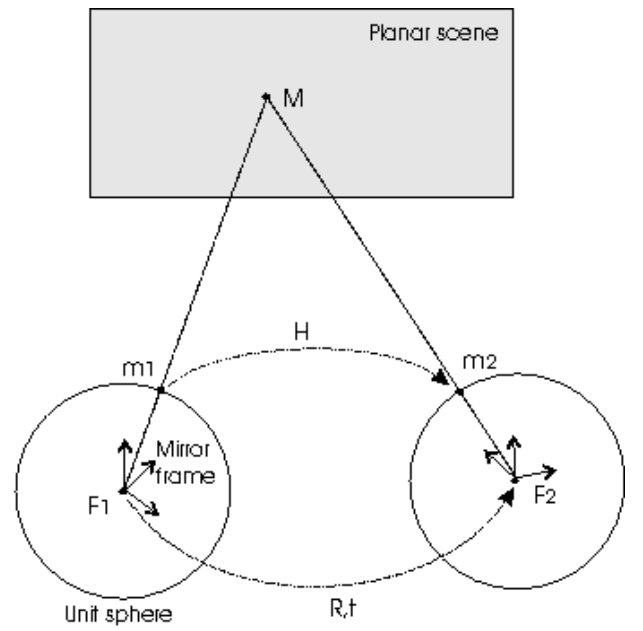

Fig. 3. Homography from two spherical projections.

\section{HORIZON DETECTION ALGORITHM FOR CATADIOPTRIC IMAGES}

From the previous considerations, the horizon line in a central catadioptric image is equivalent to a circle on the equivalent sphere and then to a plane in $3 D$ space which partitions the sphere into two regions (sky/ground). Let $\mathcal{C}$ a circle on the sphere $\mathcal{S}$ and $\mathcal{P}$ the plane which contains $\mathcal{C}(\mathcal{C}=\mathcal{P} \cap$ $\mathcal{S})$. We can show that $\mathcal{C}$ can be determined by a single point $\left(x_{c}, y_{c}, z_{c}\right) \neq(0,0,0) \in \mathcal{B}(0,1)=$ $\left\{(x, y, z) \mid x^{2}+y^{2}+z^{2} \leq 1\right\}$ and that the equation of $\mathcal{P}$ is:

$$
x_{c}\left(x-x_{c}\right)+y_{c}\left(y-y_{c}\right)+z_{c}\left(z-z_{c}\right)=0 .
$$

Moreover, the radius of $\mathcal{C}$ is $R=\sqrt{1-\left(x_{c}^{2}+y_{c}^{2}+z_{c}^{2}\right)}$. Reciprocally, any point $\left(x_{c}, y_{c}, z_{c}\right) \neq(0,0,0) \in$ $\mathcal{B}(0,1)$ defines a single circle $\mathcal{C}$. This property demonstrates that searching a circle (except the great circles) on the sphere is equivalent to searching a point $\neq(0,0,0)$ inside the sphere.

Point $\left(x_{c}, y_{c}, z_{c}\right) \in \mathcal{B}(0,1)$ partitions sphere $\mathcal{S}$ into two regions $R_{s}=\left\{(x, y, z) \in \mathcal{S} \mid x_{c}\left(x-x_{c}\right)+y_{c}(y-\right.$ $\left.\left.y_{c}\right)+z_{c}\left(z-z_{c}\right)>=0\right\}$ and $R_{g}=\{(x, y, z) \in$ $\left.\mathcal{S} \mid x_{c}\left(x-x_{c}\right)+y_{c}\left(y-y_{c}\right)+z_{c}\left(z-z_{c}\right)<0\right\}$. Searching this point which corresponds to the horizon in the image consists in classifying the points on the sphere $\{(x, y, z) \in \mathcal{S}\}$ into two classes. These classes represent respectively the sky and the ground and the color of the points defined in RGB space is used as measure of appearance.

Let $R_{s}$ and $R_{g}$, these two classes characterized by their means $m_{s}=\left(m_{s}^{R}, m_{s}^{G}, m_{s}^{B}\right)$ and $m_{g}=$ $\left(m_{g}^{R}, m_{g}^{G}, m_{g}^{B}\right)$ and by their covariance matrices $\Gamma_{s}$ and $\Gamma_{g}$. If we consider the Mahalanobis distance given by

$$
d\left(R_{s}, R_{g}\right)=\left(m_{s}-m_{g}\right)^{T}\left(\Gamma_{s}+\Gamma_{g}\right)^{-1}\left(m_{s}-m_{g}\right)
$$

the best partitioning into $R_{s}$ and $R_{g}$ is then defined by

$$
\arg \max _{R_{s}, R_{g}} d\left(R_{s}, R_{g}\right)
$$

In our case, the problem can be formulated as follows:

we are looking for point $\left(x_{c}, y_{c}, z_{c}\right)$ which gives the maximum of,

$$
\begin{array}{ccc}
\mathcal{B}(0,1) \backslash(0,0,0) & \rightarrow \quad \mathbb{R} \\
\left(x_{c}, y_{c}, z_{c}\right) & \mapsto d\left(R_{s}, R_{g}\right) .
\end{array}
$$

Then, we can compute pitch $(\psi)$ and roll $(\rho)$ angles as follows :

$$
\begin{gathered}
\psi=\arctan \frac{-x_{c}}{\sqrt{y_{c}^{2}+z_{c}^{2}}} \\
\rho=\arctan \frac{y_{c}}{z_{c}}
\end{gathered}
$$

The maximization of (2) is developed in (Demonceaux et al., 2006) using an algorithm based on a multiscale sampling of the sphere.

\section{ADVANTAGES OF COMBINING THE TWO METHODS}

In this chapter, we present two advantages provided by the combination of the horizon and homography-based methods to correctly apply planar homography principle. 


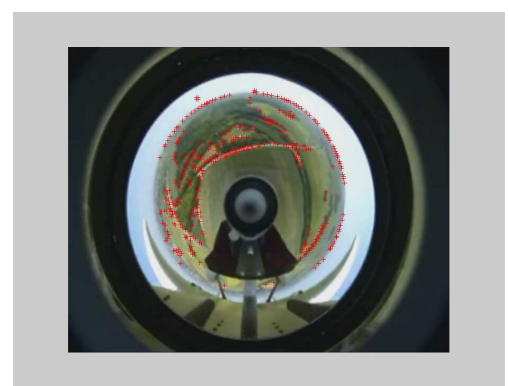

(a)

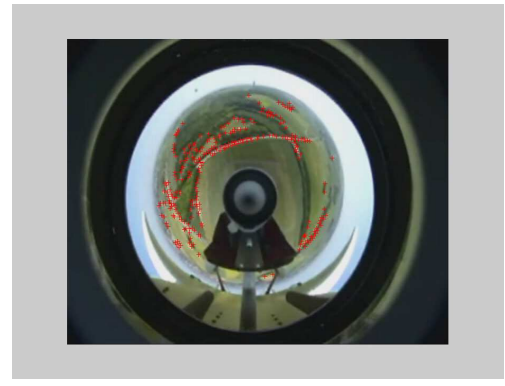

(b)

Fig. 4. By imposing horizon constraint, we can remove all the Harris features that lie in the sky or near the horizon and therefore obtain a more robust estimation of the planar homography.

\subsection{Detect inconvenient feature points}

To compute the camera motion, homography relation needs point correspondence in a pair of images. For this purpose, we have used two common tools: Harris corner detector for the features extraction part and Normalized Cross Correlation for the initial features correspondence. As depicted in figure 5 , experiments have shown that some features lie in the sky and many others lie near or on the horizon. Due to the high resemblance of these regions (sky is homogeneous and ground parts below the horizon are very similar), the correlation-based matching step for the features belonging to these regions makes a lot of inconsistent correspondences. Moreover, the points on the horizon do not actually belong to the real ground plane but rather lie at infinity. Indeed they are invariant to translation and dependent on rotation. It means they do not verify the planar homography relation and thus cannot permit to retrieve the matrix of planar homography. Therefore, it clearly appears those inconvenient features must be detected and not used for the homography estimation. Our suggestion to solve this problem simply consists in first extracting the horizon by our previously proposed algorithm and then removing all the features that lie above or near the horizon. Therefore it permits to keep only the ground features and obtain a more robust estimation of the planar homography.

\subsection{Retrieving motion parameters from the homography}

As previously explained, from the decomposition of $H$, we can retrieve the motion parameters among two set of feasible solutions by using an approximation of the normal vector $\tilde{n}=n / d$. Actually, the horizon-based method permits to calculate the normal of the horizon in the sphere space which is equivalent to the normal of the ground plane. Therefore we have a good approximation of $\tilde{n}$ and we can retrieve the motion parameters $R$ and $T$ from the homography $H$ and thus the three rotation angles.

\section{RESULTS}

Due to the fact that it is hard to attach both an INS and a catadioptric camera on our aerial vehicle, ground truth data of the UAV attitude is not available. As a consequence, we cannot qualitatively compare true data with our computed angles to date and we suggest a visual validation of the results. Experiments have shown that the estimation of roll, pitch and yaw angles correctly correspond to the evolution of the UAV along the video. In future work, we plan to work on two different aspects: first, get ground truth data and qualitatively measure the accuracy of our approach, and second, try to reset the error that might be accumulated by homography thanks to the knowledge of the absolute roll and pitch angles estimated by the horizon.

\section{CONCLUSION}

In this paper, we have suggested combining our previous horizon detection algorithm with the planar homography technique to solve the UAV attitude estimation problem based on catadioptric images. We have shown that the proposed approach provides two important advantages to correctly apply the homography method. First the horizon extraction permits to get rid of an important number of point features that do not verify the planar homography relation and thus estimate the homography more robustly. Secondly, we have explained that computing the horizon in the equivalent sphere provides an estimation of the normal vector of the ground plane which enables to retrieve the correct motion from the four solutions obtained by homography.

\section{REFERENCES}

Barreto, J. P. (2006). A unifying geometric representation for central projection systems. Comput. Vis. Image Underst. 103(3), 208217. 


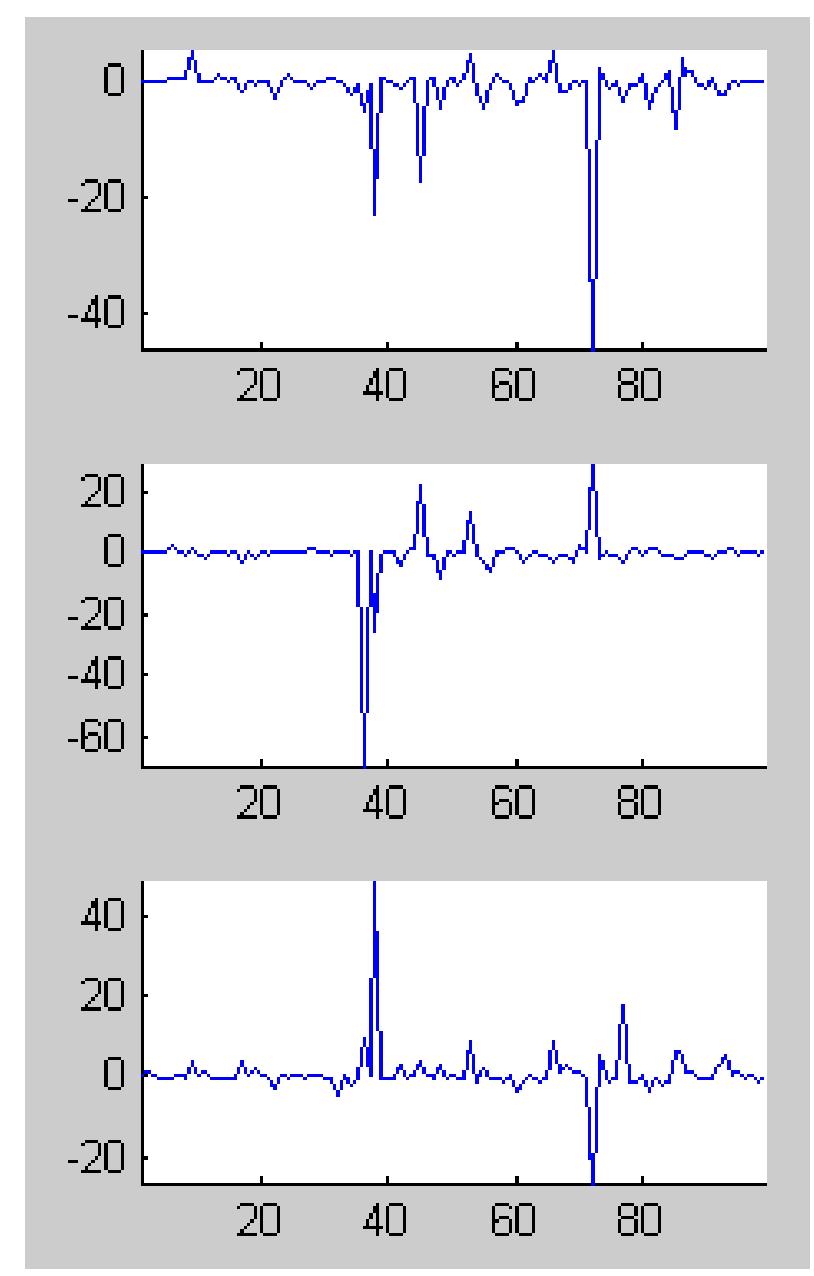

(a)

Fig. 5. Evolution of pitch, roll and yaw angles by planar homography in UAV catadioptric images. The brief jump corresponds to unreliable results..

Benhimane, S. and E. Malis (2006). A new approach to vision-based robot control with omni-directional cameras. In: Proc. of Int. Conf. on Robotics and Automation (ICRA'06). Orlando, USA.

Demonceaux, C. and P Vasseur (2006). Omnidirectional vision on uav for attitude computation. In: Proc. of Int. Conf. on Robotics and Automation (ICRA'06). Orlando, USA.

Demonceaux, C., P. Vasseur and C. Pégard (2006). Robust attitude estimation with catadioptric vision. In: Proc. of Int. Conf. on Intelligent Robots and Systems (IROS'06). Beijing, China.

Ettinger, S.M.and Nechyba, M.C., P.G. Ifju and M. Waszak (2003). Vision-guided flight stability and control for micro air vehicles. $A d-$ vanced Robotics 17(7), 617-640.

Faugeras, O (1993). Three-dimensional computer vision: a geometric viewpoint. MIT Press. Cambridge, MA, USA.
Geyer, C. and K. Daniilidis (2001). Catadioptric projective geometry. International Journal of Computer Vision (IJCV) 45(3), 223-243.

Hartley, R. I. and A. Zisserman (2004). Multiple View Geometry in Computer Vision. second ed.. Cambridge University Press, ISBN: 0521540518.

Naval, P.C., M. Mukunoki, M. Minoh and K. Ikeda (1997). Estimating camera position and orientation from geographical map and mountain image. In: 38th Research Meeting of the Pattern Sensing Group, Society of Instrument and Control Engineers.

Rushant, K. and L. Spacek (1997). An autonomous vehicle navigation system using panoramic machine vision techniques. In: Technical report CSM-298.

Wang, L.K., S.C. Hsieh, E.C. Hsueh, F.B. Hsaio and K.Y. Hunag (2005). Complete pose determination for low altitude unmanned aerial vehicle using stereo vision. In: Proc. of Int. Conf. on Intelligent Robots and Systems (IROS'05). Edmonton, Canada. pp. 316-321.

Woo, J. and I. Kweon (2005). Roubust horizon and peak extraction for vision-based navigation. In: IAPR workshop on Machine Vision Applications. 